\title{
Advancing Service Operations: The Changing Role of Industrial Engineering
}

\author{
Farayi Musharavati* \\ Department of Mechanical and Industrial Engineering, Qatar University, P.O.Box. 2713, Doha, Qatar
}

\begin{abstract}
Service operations are currently at the hub of economic activities in some parts of the world. Although indicators seem to suggest the existence and future dominance of service economies, such economies will only be sustainable if appropriate use of scientific and engineering methods are applied to the complete spectrum of service operations; i.e. the engineering aspects and the business side (management) of operating systems. The challenge, therefore, lies in selecting appropriate scientific and engineering tools, techniques, methods and technologies for implementation in those critical areas that can leverage dynamics in service operations as they are known today. Of all engineering disciplines currently available today, industrial engineering and its related equivalence or variants is among the most multifaceted disciplines that can face up to the challenge of advancing service operations to higher dimensions. Such advancement requires appropriate demonstrable, practical and transferable research results as well as suitable case studies on service operations. In light of the quest to advance service operations, this paper discusses the changing roles of industrial engineering and its crucial input in advancing service operations towards growth, development and perhaps global dominance of service economies.
\end{abstract}

Keywords: Service economy; Industrial engineering; Industrial management; Service operations; Manufacturing operations

\section{Introduction}

Traditionally, industrial engineering has been concerned with the analysis, design and control of materials, work and information in a variety of systems $[1,2]$. This traditional focus has been on 'industrial systems', in particular "hardcore manufacturing". In this mindset, the major role of industrial engineering has been to provide mathematical modeling and computer simulation tools that were lacking in the premass production eras when manufacturing was considered to be more of an art than a science. Over the years, abundant evidence and success stories that relate to increasing importance of industrial engineering in the manufacturing sector have been documented. However, the past two decades have seen fundamental shifts, in some countries, from "hardcore manufacturing" to "service operations" [3]. Although this shift and its effects have not been thoroughly investigated and evaluated, indicators show a real drive towards service operations. With this shift, comes number of questions some of which have no answers even today. For example, does this shift imply the inevitable replacement of "hardcore manufacturing" with service operations in playing a significant role as the main platform for economic growth and development? If so, what has been the impact of this shift and should other parts of the world follow suite? If not, what are the obstacles and how can these obstacles be avoided? Irrespective of answers to the above questions, the big question of interest in this paper is 'how can service operations be advanced to scale heights that are necessary for a global dominance of service economies?

The following sections of this paper attempt to position the role of industrial engineering in advancing service operations. The objectives of the present paper are: (a) to explain the role of industrial engineering, (b) to position industrial engineering in a world that seems to drift towards service economies, and (c) to describe the role of industrial engineering in advancing the state of service operations. Perspectives and opinions on the changing roles of industrial engineering will be discussed. Firstly, an explanation of the various terms that will be used in the discussions are provided. Secondly, a parallel discussion of the rise of the manufacturing sector in the wake of a global shift towards service economies is presented. Thirdly, the changing roles of industrial engineering and its influence on the shift towards service operations are described. Fourthly, an emphasis on why service operations need to strike the balance between industrial engineering and "management" is outlined. Finally, concluding remarks are discussed and pending issues in service operations highlighted.

\section{Working Terms}

Currently, the public literature lack unified definitions of some of the terms pertinent to the discussions in this paper. In order to proceed with the discussions, explanations of the various key terms will be presented.

Most discussions on economic growth and development revolve around agriculture, manufacturing and services. This paper identifies three basic platforms for economic growth [4], namely; (1) extractive industries, for which agriculture, mining, forestry and fishing are the prominent examples, (2) goods producing sector for which prominent examples are manufacturing and construction, and (3) services operations. For simplicity, these basic platforms will be referred to as: agriculture, manufacturing and service operations.

\section{Agriculture}

Agriculture can be viewed as the cultivation of animals, plants, and other life forms for food, fiber, and other products used to sustain life [5]. Historically, agriculture was the key development in the rise of human civilization in which agricultural products were used to nurture

*Corresponding author: Farayi Musharavati, Assistant Professor, Department of Mechanical and Industrial Engineering, Qatar University, P.O.Box. 2713, Doha, Qatar, Tel: +974 4403 4325; E-mail: farayi@qu.edu.qa

Received May 03, 2012; Accepted July 05, 2012; Published July 09, 2012

Citation: Musharavati F (2013) Advancing Service Operations: The Changing Role of Industrial Engineering. Ind Eng Manage 1: 102. doi:10.4172/2169-0316.1000102

Copyright: ( 2013 Musharavati F. This is an open-access article distributed unde the terms of the Creative Commons Attribution License, which permits unrestricted use, distribution, and reproduction in any medium, provided the original author and source are credited. 
the development of civilization. An agricultural-based economy is an economy for which the fundamental basis of growth and development is based on how prosperous the agriculture of that economy is. From ancient history, for example the ancient Mesopotamia, surplus from agriculture created jobs for storage and trading thus making the economy richer [6]. It can then be inferred that even in the earliest forms of civilizations service jobs were apparent in the form of storage and trading when the harvest was more than what a society needed. Modern forms of agricultural-based economy include most African countries, some parts of Asia and some parts of Latin America. Therefore, an agricultural-based economy can be viewed as one in which the highest percentage of economic growth comes from agriculture.

\section{Manufacturing}

In simple terms, manufacturing is the use of machines, tools and labor to produce goods for use or sale. Early forms of manufacturing included human activities such as craftwork. Later forms of manufacturing include advanced technologies as applied to industrial production. In the later forms manufacturing is viewed as those activities that progressively add value to raw materials in a bid to create high value products for use or for sale [7]. In this regard, a manufacturing-based economy is one that uses machines, tools and labor to make products for use or for sale. Therefore, a manufacturingbased economy can be viewed as one in which the highest percentage of economic growth comes from manufacturing. A prominent example of a manufacturing-based economy is China.

\section{Services}

It can be observed that any agricultural-based economy i.e. an economy that is producing agricultural products in excess (hence for use and selling), will need service activities in either storing the surplus or trading the surplus. The same can be said for manufacturing. Therefore, a certain degree of services (service occupations/jobs/activities) often follows success in agriculture and manufacturing. Success for both agriculture and manufacturing means producing in excess. Therefore, a service-based economy can be viewed as one in which the highest percentage of economic growth comes from service operations. Such service operations include; service companies and service packages of different magnitudes. In this regard, service operations range from providing a service to an individual to servicing the global economy while service systems are organizations designed to deliver services that satisfy the needs and wants of the organization's customers.

\section{Wide Disparities in Manufacturing Operations}

In order to address some of the questions raised in the previous sections, it is important to go back in time and track the events that lead to the success stories of the manufacturing sector as a fundamental basis for economic growth. We start our journey in Africa, a continent which has been gifted with natural resources required for agriculture and manufacturing. Despite an abundant source of natural resources, economies in Africa have not yet realized substantial growth at global levels. For example, industries in Africa account for just $1 \%$ of the world's manufacturing value added compared to $8 \%$ in Asia (excluding China) and 5\% in South America [8]. Besides the highly developed industrial base in South Africa many countries in Africa that are endowed with natural resources have the potential to attain industrialized status. Albeit, the paradox is that to date Africa, has experienced stinted industrial growth. The actual reasons for this anomaly are not clear and neither are they well understood. One of the drawbacks probably lies in inappropriate production and manufacturing polices methodologies, strategies, techniques and technologies that can be rightly implemented to leverage the situation.

Although appropriate methods and techniques are scattered all over a long list of journals, magazines, and technical papers nothing positive seems to be happening in African economies. This situation leaves a lot to be desired and people will always ask why. Two issues can be attributed to this anomaly: (a) the volumes of research work in journals and research papers overwhelm practitioners. This leaves practitioners without proper guidance and practice for implementation. What is required to redeem this situation are not just general methodologies, strategies, techniques and technologies as is found in the public and private literature. Proven methodologies, strategies, techniques and technologies developed to address the unique manufacturing landscape prevailing in Africa may be a good starting point; (b) a drawback for industrial engineering is that "management"sometimes over shadows industrial engineering particularly in service operations.

For the discussions in this paper "management" is highlighted in order to differentiate general management and industrial management for which the later is an essential component of industrial engineering. Thus management in this paper refers to the process of planning and decision-making, organizing, leading and controlling an organization's human, financial, physical and information resources in an efficient and effective manner $[9,10]$. In other words most service operations particularly those in the small-to-medium category do not realize the need and importance of industrial engineers in their operations. Instead they prefer to hire a manager rather than hire an industrial engineer. This has a negative effect on the performance of the operations since the service operation will lack the innovation, creativity and ingenuity that industrial engineers can bring to various operating systems.

From the examples discussed in the previous paragraph we learn a few issues that need to be addressed: (1) general knowledge and information provide little help to practitioners. As a result, focused and time tested knowledge and information supported by a wide range of case studies inclusive of all global scenarios are required; (2) for knowledge and information to redeem peculiar situations, such knowledge must address the uniqueness of the situation at hand. Although a comprehensive analysis of the factors that define the uniqueness of the various situations is outside the scope of this paper, a few observations can be noted. For example, most public databases usually contain contemporary issues dealing with high technologies advanced and recent technologies that may not be specifically relevant for other situations. In addition, situations may be dynamic in such a way that what is 'currently' called unique may not be unique 'tomorrow'. In such cases dynamic knowledge and information data bases are required in order to perpetuate the operations; (3) such knowledge and information must be available and accessible to the public. Simple as it may seem, crucial results, ideas, implementations and success stories are usually paraphrased and thus hidden to the public or they may be disclosed only in private databases. In this regard open access initiative which allow users a free, irrevocable, worldwide, perpetual right of access to knowledge and information can play an important role in redeeming situations; (4) such knowledge and information must be easily transferable. For example, in most cases engineers struggle to understand the very basics of presented methodologies, techniques and technologies mainly because of the wide disparities between the available technologies, processes and systems. This is because most sources of knowledge always emphasize cutting-edge research, recent, new and advanced technologies in advanced processes and systems. Such information leaves engineers in less industrialized operations without 
guidelines on how to improve their operations. For those engineers in industrialized regions, the rapid developments and available case studies leave them equally perplexed due to the overwhelming volumes of new and innovative discoveries. If the issues mentioned above are addressed properly in a well-organized source of information, it may be possible to redeem the various scenarios cited above.

\section{Service Operations - The Curse of "Management"}

The contribution of manufacturing to the overall economic activity in any region of the world (less industrialized, industrialized or more industrialized) cannot be denied. Neither can the current shift towards service economies be brushed aside. The shift towards service economies has been partially due to competitive global markets and other global issues and activities. Global competition has changed the whole production scenario. Companies have suddenly find themselves in a global economy that demands higher levels of productivity, versatility, flexibility and re configurability in operating systems. These levels make the difference between less growth and development and more growth and development. The real question is: in the current global scenarios, is it still possible to redeem the stinted growth in less industrialized regions of the world in order to improve the low manufacturing value added figures? If so, then, how do such "redeemed situations" relate to the current shifts towards service economies? And most important of all, how can service operations be advanced to scale the highest possible heights? In order to answer some of these questions we proceed with our journey from Africa to India.

India is one of those economies that have been trying in the past two decades to shift from an agricultural and hardcore manufacturing economy to service operations. A prominent example of this is the perceived boom in information technology, software, telecommunication services and business process outsourcing [11]. For some time, it was believed that information technology, software, telecommunication services and business process outsourcing in India will overwhelm the world and result in significant economic growth and development at global scale. Although the potential for a service economy is still available and a lot of benefits have been accrued, of late concerns for Indian economy have resulted in the need to revisit the agricultural sector. The reason for this concern came as a realization that despite success and growth due to service operations, large regional income disparities still exist in India [12]. Hence indicators for a revisit of the fundamental time-tested and permanent basis for economic growth and development; i.e. agriculture and manufacturing, are indeed the right direction to follow. Could this suggest that India has recognized that for a service economy to be stable and sustainable, it must be backed by a flourishing agriculture and/or hardcore manufacturing base? Or is the opposite true? Like any other paradox, the real reasons for this situation are not clear and the dynamics are not known neither are they clearly understood. What, if any, could have been missing in India's quest for a service economy? And more important, what is the difference between India's purported venture towards a service economy and other nations like the US that seem to be thriving on service operations?

Perhaps the way forward in addressing the issues highlighted in the previous paragraph can be revealed by going back to the earlier times and track US's success towards a formidable service economy. US started its journey towards a service economy from an agricultural economy [4]. With time, manufacturing experienced a boom and the US economy automatically became dominated by manufacturing in the early part of the twentieth century. This does not necessarily mean that the US abandoned agriculture but it was a mere shift in terms of percentage growth rates accrued through manufacturing. For some time, the US economy thrived on agriculture and manufacturing. However, history shows that as the US economy was depending on agriculture and manufacturing, the services required to support these two agents of economic change were slowly becoming a significant contender for growth and development. Hence, today there is clear evidence that service operations have overtaken both agriculture and manufacturing in the US economy.

Albeit, it cannot be denied that US started off with an agricultural economy, followed by 'hardcore manufacturing' as prominent basis for economic development. A number of successful cases, stories, research papers, and technical papers of how the US economy struggled to reckon with say Japanese methodologies, techniques and technologies in fierce competitions for global market shares have been reported. In addition, the US economy also went through a number of incrementally iterative revolutions in various production operations notably in hardcore manufacturing. However, over the years, lessons learnt, knowledge and information accrued helped the US industry to progressively develop and advance to the highest scales in manufacturing prior to a focus on service operations. Such historical accounts, show that there is no 'short cut' to growth and growth moves from one step to another otherwise we risk the danger of stinted growth unless some catalytic factors are injected into the quest for economic growth and development. Most important of all is the observation that the growth and rise of US towards a service economy went through more or less the same difficulties, uncertainties and explorative ways similar to those encountered along the manufacturing journey towards supremacy. Failures, mistakes, difficulties and challenges encountered throughout the manufacturing journey helped in shaping the US economy to its current status. This has also been true for the US service industry.

Other questions that may arise due to the previous discussions are: where do economies like Qatar and most of the gulf countries that rely on exceptional natural resource (i.e. without a significant agricultural and hardcore manufacturing base) stand? What about nations such as Singapore that even struggle to get raw materials for the building and construction industry? What about China and its seemingly rapid growth towards a hardcore manufacturing boom? And more important, what is it that propelled and perpetuated the US economy towards a seemingly dominant service economy?

To summarize, history tells us that countries with successful agriculture and/or manufacturing histories are the ones that now have the ability to continuously create large service operations in which more than $50 \%$ of revenue comes from services rendered and/ or provision of service packages [4]. As has been discussed earlier on, a strong agricultural base automatically creates service operations that are required to support agriculture. Equally true is the fact that a strong manufacturing base creates supporting service operations. In both cases, however, the support service operations are only a fraction of the services required in further processing or trading of surplus agricultural products or surplus manufactured goods. This means that countries in Africa, Asia or Latin America that are still depending on a 'weak' agriculture base (i.e. those that produce just what is needed locally) will have to focus first on producing more surplus in order to realize significant growth in service operations. As for India, the potential for developing a service economy exists since it has already been demonstrated [13]. Maybe the way forward for India would be to revisit either agriculture or manufacturing and maintain excellence in 
these sectors while concurrently pursuing the move towards a service economy.

The earlier boom in the US manufacturing sector could have been partially due to the quick realization and adoption of appropriate methodologies, techniques and technologies required to advance the manufacturing sector from an earlier 'curse' of manufacturing as an art to the 'revelation' of manufacturing as a science. Manufacturing as a science requires scientific methods and approaches in order to advance. Like earlier views of manufacturing as an art, most service operations today are carrying the 'curse' of "management" without the right blend with the so much needed industrial engineering. Based on these perspectives, a service economy can realize faster significant growth if it is backed by either a prosperous agricultural base or a strong manufacturing base. Like the time-tested advances in manufacturing operations, service operations can advance and scale the heights if appropriate scientific tools, techniques, methods, and technologies are developed and implemented. Although the issues mentioned above seem to be obvious to any technical mind, what may not be realized is that industrial engineering is one of the greatest mysteries behind the progressive growth of both the manufacturing and service operations in the US economy. Some may argue that industrial engineering has always been there and so why does it seem to be one of the major agents of change in either manufacturing and/or service operations? One of the answers to this question lies in the changing role of industrial engineering. These changing roles were also reciprocated by appropriate and timely changes in university curriculum on industrial engineering and its equivalent variants.

\section{The Role of Industrial Engineering}

Most African economies have failed to realize significant growth in both the agricultural sector and the manufacturing sector. The reason for this failure could, among others, be that "management" takes precedence and overshadows industrial engineering in most operations. As long as the right balance between "management" and industrial engineering is not adopted or sustained, it is difficult to realize and/or sustain significant economic growth and development [14].

A critical examination of most service operations, in particular the small-to-medium operations reveals that "management" almost always over shadows industrial engineering. This may seem to be a myopic problem of decision makers in service operations but the crux of the matter lies in that traditional engineering has been associated with mechanical engineering, electrical engineering, civil engineering etc. These engineering disciplines seem to be so much divorced from service operations. Hence most decision makers for human resources in service operations see no point in engaging the services of industrial engineers. This is particularly true in small-to-medium service and manufacturing operations. Although some service operations have realized the importance of industrial engineering, some of the decision makers in service operations are probably not convinced or simply not aware of the fact that industrial engineering is a crucial ingredient to their operations [15].

\section{What is Industrial Engineering?}

For many years a number of authorities have tried to define and explain to the public what industrial engineering is all about. Typically, it has been generally agreed that industrial engineers use computer simulation, extensive mathematical tools and modeling, as well as computational methods for system analysis, evaluation, and optimization [16]. This view of industrial engineering made a significant contribution to the US economy during the mass production eras [17]. In this era the US, with the help of some Japanese concepts, techniques and methods realized and achieved significant growth and development. After this era, it was noticed that in general industrial engineers work to make people's lives better [18]. This new spectrum appraised industrial engineers as engineers that design systems that integrate people, information, materials, and equipment in order to provide services and manufacture products by the most efficient, productive, and safest methods [16,17]. This new perspective on industrial engineering introduced prominent aspects on designing manufactured goods and services. From this stage onwards, a number of significant changes in the roles of industrial engineers started to spring up. These changing roles were actually tailored to dynamic changes in the requirements of various types of production systems. The idea in this case was to, tailor make all engineering disciplines and allow the roles to evolve with the changing demands in various industries. Therefore, one of the prominent factors that may be instrumental in retarding operations (in services, process manufacturing or discrete manufacturing) is the failure to recognize and hence employ the dynamic and incremental roles of industrial engineering in operating systems. The following sections discuss some of the changing roles of industrial engineering and how they have affected the growth and development of various types of industries.

\section{The changing role of industrial engineering}

Most of the obvious roles of industrial engineering in the manufacturing cannot be disputed. However, the changing roles of industrial engineering in service operations have not been fully recognized and hence have not been fully utilized. This is mainly because the term service operation has been misunderstood by some organizations, practitioners and management of service operations. Examples of commonly known service operations include: food services i.e. restaurants (fast food or otherwise); grocery services such as supermarkets, and departmental stores; business services such as consulting, banking and financial services; trade services such as retailing, maintenance and repair; social/personal services such as healthcare and recreation; public services such as government and education; and infrastructure services such as transportation and communication systems. From this general list, it is very difficult to visualize the role of industrial engineering. However, it is fairly easy to imagine the role of "management" since it is easier to imagine management roles and skills in most operating systems even the smallto-medium range [19]. This is the reason why "management" may over shadowed industrial engineering thus marginalizing the rapid growth and development of service operations. Moreover, since industrial engineering was formerly associated with what is generally perceived to be 'industries', examples of service operations mentioned above may not realize the role and importance of employing an industrial engineer. This is even more difficult for small-to-medium service operations, in particular those that are found in less industrialized regions of the world.

One of the most undisputed roles of industrial engineering is the analysis, design and development of 'systems'. This is the reason why systems simulation is such an important tool for industrial engineers. A close examination of some service operations reveals three major components: (1) service supporting facility, (2) facilitated "goods" and (3) the service itself [4]. While the facilitated 'goods' and the service itself may be one in some service operations, the breakdown above can be used to illustrate the significant roles of industrial engineering in service operations. Depending on the type of service operations, the 
service support facility may or may not be visible to the customer (consumer). For example, if a restaurant has dining facilities then the dining support facility will be visible to the customer. In this case, the design of layout and operations of the dining facility becomes part of the customer's perception of quality of service offered. However, in a fast food restaurant, dining facilities do not come into the picture. The layout (e.g. drive-in or walk-in) as well as operational aspects in delivering the service become major proponents of the customer's perception of quality of service. In the case of a restaurant, the facilitated "good" may be a piece of chicken or some french fries. In such a case, the customer's perception of service will incorporate, among others, the taste of the facilitated goods as well as the way in which the service is delivered [20].

In the example described above, the role of the industrial engineer would be to analyze, design/redesign and/or optimize the service system. Although it may sound trivial, it is important to identify the service system in a service operation [21]. For a restaurant, the service system is composed of the equipment used in the restaurant kitchen, the layout of the restaurant kitchen, layout of dining areas, the layout of the drive-in or walk-in procedures, and the procedures used to provide the service and maintain quality and delivery standards. It is clear that all the components in the service operations do not require a manager, but an industrial engineering. Paradoxically, the situation on the ground actually prefers managers and operators to handle issues and problems in the service system. While this situation has prevailed to date, it is worthwhile to re-consider the ingenuity of an industrial engineer in these matters since an industrial engineer is equipped with both engineering and management skills.

Industrial engineering or "management": One of the issues highlighted in the previous sections was that in service operations "management" overshadows industrial engineering. In order to strike the correct balance between industrial engineering and "management", it is important to appreciate the differences in these seemingly intersecting disciplines. Albeit, industrial engineering differs from "management" in the sense that industrial engineering emphasizes more on modeling, analysis, organization, and design of operations and processes in any given production system including service operations. On the other hand, "management" focuses more on the functioning and the business side of operations [22]. Although industrial engineering has some industrial management components, such components focus more on the operations engineering side. Thus, the role of industrial engineers can be viewed as those roles associated with resolving system design and industrial management issues that prevent organizations from achieving peak performance. In this view, solutions are arrived at through rigorous quantitative analysis and have significant financial impact. Although overlaps exist (which is one of the reasons why "management" overshadows industrial engineering) industrial engineering usually emphasizes more on extensive mathematical proficiency and usage of quantitative and scientific methods. A distinguishing focus in industrial engineering has been the integration of humans, machines, and materials to achieve optimum performance in operating process systems. Methods of operations research and ergonomics provide the fundamental tools for performing operations analysis and process system integration. More recently, the field of industrial engineering has expanded to include nonindustrial operations involving supply, distribution, transportation, communication, information handling, medical care and safety [23]. The design and control of these systems requires the use of scientific and engineering methods in a variety of research and application areas of industrial engineering. Therefore, the role of industrial engineering in advancing service operations can never be overemphasized. In the following subsections, discussions on some of the specific changing roles of industrial engineering with respect to changes in operations requirements are presented.

Universality of industrial engineering: Unlike most disciplines, industrial engineering is versatile in its applicability. Examples of areas in which industrial engineering can play a significant role includes: designing an assembly workstation, strategizing for various operational logistics, consulting, developing new financial algorithms or loan systems for a bank, streamlining operation and emergency room location or usage in a hospital, planning complex distribution schemes for materials or products (supply chain management), and shortening lines (or queues) at a bank, hospital, or a theme park [24]. From these examples, it can be observed that industrial engineering can be applied in almost every industrial sector. Core disciplines in industrial engineering include; statistics, operations research, economic decision making, human engineering, and industrial management [25]. In addition, industrial engineering problems are found in retail, distribution and supply chain, banking, healthcare, and many other environments. It can, therefore, be observed that modern industrial engineers are trained to understand both the technical and business sides of operations. This universality of industrial engineering makes industrial engineers effective professionals in a global economy.

Parallelism in the changing roles of industrial engineering: Since inception, the roles of industrial engineering have evolved in tandem with the developments in industry. In the pre-mass production era, industrial engineering was primarily involved in human performance at work places, in addition to engineering economics, production planning, facilities planning, and quality control. In the post mass production era, it was observed that two main aspects were going to affect and influence the future of the manufacturing sector. These two aspects were identified as (1) mathematical modeling, and (2) computers. In order to address specific issues arising due to trends in manufacturing sector during the post mass production era, more rigorous approaches to mathematical modeling and use of computers were required. This need resulted in the shifting of focus in industrial engineering training curriculum from the traditional skills and techniques into new techniques such as mathematical programming, simulation, and computer data processing. This focus expanded the role of industrial engineering in operating systems.

However, problems always arise when shifts occur. Some scholars may take a 'die hard' point of view, which argues that a shift in focus is effectively a dilution in the industrial engineering roles. Some scholars may accept the shift as a progressive development while others may remain on the brink. Albeit, genuine shifts are necessary since they usually represent the difference between operations advancing to higher levels and static or retarding operations. Thus, by taping the useful information implied in a shift, it is possible to advance beyond current operating levels in service operations. On the other hand, failing to realize such and similar shifts often results in stinted growth from which only catalytic factors are required in order to make-up the time lost due to failure to recognize fruitful shifts.

In addition to shifting focus, the number of industrial engineers required in operating systems was also increasing due to the continued expansions, growth and developments in the manufacturing sector. While the manufacturing sector was moving towards operational excellence, service operations were also on the rise in the 1970s. Since the influence and contribution of industrial engineering had been documented, it was time to employ industrial engineers in service 
operations. Like in the manufacturing sector, industrial engineering was initially brought into service operations for the fundamental purposes of analyzing human performance at work places, human motivation, engineering economics, production and facility planning, and quality control issues. However, as the roles of industrial engineering continued to change and evolve (in both the manufacturing sector and service operations) industrial engineering became a formidable force to reckon with in the engineering of service operations. This resulted in diversification of industrial engineering into other fields such as banking and insurance; health care; transportation and distribution; environmental protection; and information processing.

At the turn of the twentieth century, concepts such as lean and six sigma were flourishing in the manufacturing sector and there was a major drive to implement similar concepts in service systems. As a result, industrial engineering found places in implementations of lean and six sigma in most of the prominent service operations such as healthcare and pharmaceuticals. In these applications, industrial engineers work to eliminate wastes of time, money, materials, energy, and other resources in operating systems. A point worth emphasizing is the fact that industrial engineering science and technology has been with us for a long time since the introduction of scientific management principle. However, today industrial engineering theory, research and advancements to support the changing roles of industrial engineers lag behind the current needs of both manufacturing and service operations. More research results are required; in particular applications of proven principles and theories in manufacturing should be developed and adapted for implementation in service operations. Such achievements can help in realizing the growth of a global service economy that can improve the lives of many in particular developing regions of the world.

\section{Concluding Remarks}

In this paper, a number of perspectives, points of view and opinions on advancing service operations have been discussed. It has been emphasized that significant growth and development into a service economy can be sustained if it is backed by a prosperous agriculture base or a strong and thriving manufacturing base. These two factors have been identified as the backbone of service operations. As such, countries that are still struggling to make it to international markets to compete as agricultural economies or manufacturing based economies will find it difficult to enter the realms of sustainable service economies. It appears that a normal and progressive growth towards a service economy requires a path from an agricultural economy, to manufacturing based economy. Although economies that thrive on the abundance of exceptional natural resources such as oil and gas, can realize the short-cut growth to service economies, catalytic factors must be present to support such rapid growth and development. A question to think about is; what are these catalytic factors and how can they be used to jump start the journey towards sustainable service economies?

It has also been discussed that the focus on the roles of industrial engineering have shifted over the years from the traditional role that was dominated with plant layout planning to other roles of a tertiary nature in a wide variety of production operations. Over the years, industrial engineering roles have evolved and diversified in tandem to the changing needs and requirements of industrial and systems operations. In order to sustain the changing roles of industrial engineering, it is necessary to develop scientific tools, techniques and technologies relevant to the nature of current operations. In this quest, research in industrial engineering should focus on developing service operations science and technology that can advance service operation to higher scales. This will also ensure rapid developments and growth towards service economies. Although industrial engineering has a strong focus on the engineering side of operations, industrial engineering has some industrial management components as well. It has also been appreciated that general management has some technical aspects that overlap with industrial engineering. Therefore, the right balance between industrial engineering and "management" in operations must be achieved in order to leverage service operations and advance them to highest possible scales. Another question yet to be answered is; 'how do practitioners strike the correct balance between industrial engineering and "management" in either service or manufacturing operations?

\section{References}

1. Norman NB (1963) Operations Research and Industrial Engineering: The Applied Science and Its Engineering, Oper Res 11: 387-398.

2. http://ioe.engin.umich.edu/overview/index.php

3. Spohrer J, Maglio PP, (2008) The Emergence of Service Science: Toward Systematic Service Innovations to Accelerate Co-Creation of Value. Production and Operations Management 17: 238-246.

4. http://www.referenceforbusiness.com/management/Sc-Str/ServiceOperations.htm

5. International Labour Organization $88^{\text {th }}$ session (2000) Safety and health in agriculture. International Labour Office Geneva 77

6. http://en.wikipedia.org/wiki/Mesopotamia

7. Mikell PG (2009) Automation, Production Systems, and Computer Integrated Manufacturing (3rdedn), Phi Learning

8. Siddiq MA (2000) Africa Review June 2000 Industrialization-an engine for growth.

9. Morse LC, Babcock DL (2009) Managing Engineering and Technology: An Introduction to Management for Engineers. ( $5^{\text {th }}$ edn), Prentice Hall.

10. Chang CM (2005) Engineering Management: Challenges in the New Millennium. Engineering Management Journal 15: 48

11. Banga R (2009) Critical Issues in India's Service-Led Growth. Indian Council for Research on International Economic Relations.

12. http://www.altiusdirectory.com/Business/indian-economy.php

13. Vira B, James AL (2012) Building Cross-Sector Careers in India's New Service Economy? Tracking Former Call Centre Agents in the National Capital Region. Development and Change 43: 449-479.

14. Metters RD, King-Metters KH, Pullman M, Walton S (2005) Successful Service Operations Management. USA.

15. Schmenner, Roger W (1995) Service Operations Management. Prentice Hall, USA.

16. Katsundo Hitomi PE (1989) Non-mass, multi-product, small-sized production: the state of the art. Technovation 9: 357-369

17. David AH (1984) From the American System to Mass Production, 1800-1932 The Development of Manufacturing Technology in the United States. The Johns Hopkins University Press, United States of America.

18. Quan-qing LI, Ming LI (2011) Thinking about the Application and Developmen Strategy of Industrial Engineering. Industrial Engineering and Engineering Management (IE\&EM), 2011 IEEE 18Th International Conference 2125-2129.

19. Schmenner RW (1986) How can service businesses survive and prosper? Sloan Manage Rev 27: 21-32.

20. Theodore L (1972) Production-line approach to service. Harvard Business Review 41-52. 
Citation: Musharavati F (2013)Advancing Service Operations: The Changing Role of Industrial Engineering. Ind Eng Manage 1: 102. doi:10.4172/21690316.1000102

Page 7 of 7

21. McCluskey Marc (2004) How Mature is Your Service Operation? Supply Chain Management 8: 17-20.

22. Industrial Engineering and Management by Reddy (Dec 1, 2007, New Age International Pvt Ltd Publishers
23. http://en.wikipedia.org/wiki/Management_engineering

24. http://www.uta.edu/ie/?fuseaction=content\&keyname=graduateatuta

25. Taylor FW (2005) The Principles of Scientific Management. ( $1^{\text {st }}$ edn) $1^{\text {st }}$ World Library Literary Society, USA. 